\title{
A inteligência epidemiológica como modelo de organização em saúde
}

\author{
Epidemiological intelligence as a model of organization in health
}

Antonio Luiz Rodrigues-Júnior ${ }^{1}$

${ }^{1}$ Faculdade de Medicina de Ribeirão Preto,

Universidade de São Paulo Av. Bandeirantes 3900,

Monte Alegre. 14049-900

Ribeirão Preto SP.

alrj@fmrp.usp.br

\begin{abstract}
The concept of epidemiological intelligence, as a construction of information societies, goes beyond monitoring a list of diseases and the ability to elicit rapid responses. The concept should consider the complexity of the definition of epidemiology in the identification of this object of study without being limited to a set of actions in a single government sector. The activities of epidemiological intelligence include risk assessment, strategies for prevention and protection, subsystems of information, crisis management rooms, geographical analysis, etc. This concept contributes to the understanding of policies in health, in multisectorial and geopolitical dimensions, as regards the organization of services around public health emergencies, primary healthcare, as well as disasters. The activities of epidemiological intelligence should not be restricted to scientific research, but the researchers must beware of threats to public health. Lalonde's model enabled consideration of epidemiological intelligence as a way to restructure policies and share resources by creating communities of intelligence, whose purpose is primarily to deal with public health emergencies and disasters.
\end{abstract}

Key words Epidemiologic intelligence, Emergencies in public health, Healthcare networks
Resumo O conceito de inteligência epidemiológica, como uma construção das sociedades de informação, vai além da vigilância de uma lista de doenças e da capacidade em acionar respostas rápidas; tal conceito deve considerar a complexidade da definição de epidemiologia na identificação deste objeto de estudo, sem ser limitado a um conjunto de açães de um setor do governamental. São atividades de inteligência epidemiológica: a avaliação de risco, as estratégias de prevenção e de proteção, os subsistemas de informações, as salas de situação, a análise geográfica etc. Este conceito contribui com a compreensão sobre políticas na área da saúde, numa dimensão multisetorial e geopolítica, no que se refere à organização dos serviços em torno das emergências de saúde pública e da atenção básica, além dos desastres; as atividades de inteligência epidemiológicas não devem se restringir à pesquisa científica, mas os sujeitos devem atentar para as ameaças à saúde pública. O modelo de campo de saúde possibilitou a reflexão sobre a inteligência epidemiológica, como sendo uma forma de rearranjar as políticas e compartilhar os recursos, formando comunidades de inteligência epidemiológica, cuja finalidade é, essencialmente, enfrentar as emergências em saúde pública e os desastres.

Palavras-chave Inteligência epidemiológica, Emergência em saúde pública, Redes de atenção à saúde 


\section{Introdução}

A noção de inteligência epidemiológica tem sido vinculada às atividades de vigilância epidemiológica $^{1,2}$. Há uma distinção entre atividade de inteligência em epidemias, do inglês epidemic intelligence, e atividade de inteligência epidemiológica, ao se considerar que o objeto de estudo da epidemiologia engloba o conjunto de eventos, ou condições, que têm influência sobre o estado de bemestar de pessoas e de populações, incluindo as epidemias.

Define-se inteligência estratégica como o "conjunto das ações coordenadas de busca, tratamento e distribuição, para uso, da informação útil para os atores econômicos", cujas ações "são feitas dentro da lei com todas as garantias para a proteção do patrimônio da empresa, nos melhores prazos e custo"3; a inteligência empresarial, ou competitiva, representa um tipo de atividade direcionada para a produção de informações de mercado e de inovações, cuja antecipação de decisões garante a subsistência de organização ${ }^{4}$; o Decreto no 4.376, de 2002, define "inteligência" como "atividade de obtenção e análise de dados e informações, e de produção e difusão de conhecimentos, dentro e fora do território nacional, relativos a fatos e situações de imediata ou potencial influência sobre o processo decisório, a ação governamental, a salvaguarda e a segurança da sociedade e do Estado"; por sua vez, inteligência epidemiológica resulta da evolução das sociedades de informação em saúde, em consonância com o modelo epidemiológico de campo de saúde de Lalonde, por exemplo.

\section{Ciclo de informação, inteligência e vigilância em saúde}

O processo de produção de informação é formado pela coleta de dados, pelo tratamento, pela análise e pela distribuição da informação, que é conhecido como ciclo de informação. A informática contribui com a agilidade dos ciclos de informação - criando aplicativos de coleta e de gerenciamento de dados - e com a transformação de coleções de dados em algo que seja de interesse estratégico. $\mathrm{O}$ ciclo de inteligência pode ser concebido como um processo que admite ciclos de informação, acrescido de análise de risco (percepção, concepção, gerenciamento e comunicação), de tomada de decisão e de ação $0^{5}$. Tarapanoff et al. ${ }^{6}$, além de admitirem a existência de subsistemas de informação e de inteligência, consideram que não existem atividades de inteligên- cia sem existirem ciclos de informação estruturados. A análise de risco é uma importante ferramenta para o gerenciamento de crises, de desastres, de emergências, de epidemias etc. Esta análise pode ser quantitativa ou qualitativa, sendo a pesquisa operacional essencial para a elaboração de estratégias.

Definir atividade de inteligência não é fácil, mesmo sendo este um ofício antigo ${ }^{2}$, remetendo, em ato reflexo, às ações dos serviços secretos ligados à segurança nacional e que são reconhecidas como uma "ferramenta de poder". Cepik ${ }^{7}$ destaca a seguinte dualidade: "inteligência é toda informação coletada, organizada ou analisada para atender à tomada de decisões", que resulta das sociedades de informação, numa abordagem estratégica; e "inteligência consiste em coletar informações sem o consentimento, sem a cooperação ou mesmo sem o conhecimento por parte dos alvos", que é desempenhada pelo núcleo coercitivo do Estado, com finalidade investigativa, numa abordagem restritiva. Por um lado, tal dualidade constitui contraposições capazes de dividir opiniões, estigmatizando a temática, mas, por outro lado, pode contribuir com o debate coletivo e com a epistemologia.

Apesar desta dualidade, as atividades de inteligência podem ser classificadas de acordo com a morfologia da fonte de informação que utilizam. Dentre os vários tipos, a fonte humana é a mais tradicional. Reconhecida pela abreviatura "humint", do inglês "human intelligence", é caracterizada pelo conteúdo de prontuários, questionários, cadastros, ensaios clínicos, experimentos etc., além de caracterizar o informante (população) da equipe do Centro de Informações Estratégicas da Vigilância em Saúde (CIEVS) sobre fatos atípicos que contribuam para a detecção de emergências em saúde pública. Outro tipo de fonte é caracterizado pela codificação de dados, como o Código Internacional de Doenças, e pela criptografia dos bancos de dados, que garantem a confidencialidade dos arquivos de dados (SINAN, SIM, SINASC, SISCEL etc.) e a segurança da comunicação entre computadores em rede, que é reconhecida pela abreviatura "sigint", do inglês "signal intelligence". Há, também, as fontes baseadas em imagens, que são reconhecidas pela abreviatura "imint", do inglês "imagery intelligence", que gerenciam bitmaps provenientes de sensoriamento remoto, fotografias, lâminas microscópicas, microscopia eletrônica, radiografias, tomografias etc. Há fontes que administram certificações e autenticações, que servem para atestar a veracidade de documentos, comunicações, bio- 
metria e registros, que caracterizam atividades de "mensuração" e de "assinatura", reconhecidas pela abreviatura "masint", do inglês "measurement and signature intelligence". Finalmente, a abreviatura "osint", do inglês "open source intelligence", que identifica fontes públicas de distribuição de informação, que provê acesso a documentos oficiais, livros, revistas, relatórios, bancos de dados etc., de maneira ética e legal, como os portais da Biblioteca Virtual em Saúde, do Instituto Brasileiro de Geografia e Estatística, do Departamento de Informática do SUS (Datasus), dentre outros.

A atividade de vigilância epidemiológica, como ponto de partida para a formação de sociedades de informação epidemiológica, numa abordagem estratégica de atividade de inteligência, pode ser definida como uma "observação contínua da distribuição e [das] tendências da incidência de doenças mediante a coleta sistemática, a consolidação e a avaliação de informes de morbidade e mortalidade, assim como de outros dados relevantes, e a regular disseminação dessas informações", sendo "olhos e ouvidos da

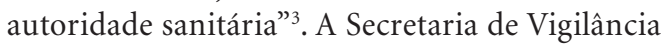
em Saúde (SVS) é o órgão responsável por desenvolver a vigilância epidemiológica, cuja estrutura inclui um subsistema de informação - que tem o objetivo de investigar doenças de notificação e de quantificar sua incidência - e um subsistema de inteligência, que participa da elaboração de políticas públicas, das ações específicas, da indução da pesquisa e da distribuição de informação estratégica e emergencial. O aperfeiçoamento do subsistema de informação representa a melhoria contínua dos ciclos de informação, com o desenvolvimento dos sistemas de coleta, da arquitetura da informação, da capacitação de profissionais e da formação de sociedades da informação. O desenvolvimento do subsistema de inteligência depende de interações verticais e horizontais entre "agências especializadas", do compartilhamento de responsabilidades, de recursos e de políticas, com vistas à tomada de decisões e ao desencadeamento de ações conjuntas.

\section{Inteligência epidemiológica}

Originalmente, a atividade de inteligência, como um ramo do conhecimento estratégico, foi desenvolvida pelas comunidades militar e judiciária; destes âmbitos, deriva uma bagagem conceitual relevante, que pode ser considerada na construção de um conceito de atividade de inteligência compatível com os interesses coletivos, que seja direcionada para o aperfeiçoamento das ações de proteção do estado de bem-estar de pessoas e de populações, e que sejam fundamentadas em valores éticos e legais.

As atividades de inteligência são focadas em objetivos, ou objetos, ou simplesmente alvos, como, por exemplo: o domínio de tecnologias, o acesso a informações privilegiadas, um patrimônio, um segredo ou qualquer objeto que tenha valor estratégico. No caso de atividades de inteligência epidemiológica, o objeto/objetivo/alvo pode ser a proteção do estado de bem-estar de populações. O objeto pode ser ameaçado por evento ou comportamento indesejados; o estado de bem-estar é ameaçado pelas epidemias, pelos desastres, pela falta de organização dos serviços, pelas emergências em saúde pública, como definidas no Regulamento Sanitário Internacional.

O Decreto no 4.376 de $2002^{8}$ define atividade de contrainteligência como aquela "que objetiva prevenir, detectar, obstruir e neutralizar a inteligência adversa e ações de qualquer natureza que constituam ameaça à salvaguarda de dados, informações e conhecimento de interesse da segurança da sociedade e do Estado, bem como das áreas e dos meios que os retenham ou em que transitem". São reconhecidas como atividades de contrainteligência epidemiológica as ações que defendam a integridade do estado de bem-estar, como a eliminação de reservatórios naturais de doenças e dos complexos patogênicos, os programas de imunização, os contratos de gestão do SUS, o aperfeiçoamento do subsistema de informação, a proteção do conhecimento, os programas de biossegurança e de biosseguridade, os planos de contenção etc. Estas atividades podem ser efetivadas por políticas públicas ou por ações isoladas.

As atividades de contrainteligência epidemiológica podem ser desenvolvidas por medidas de defesa ativa, que são aquelas baseadas na detecção e na neutralização da ameaça, como um agente etiológico. A Unidade de Respostas Rápidas (URR) do CIEVS tem as tarefas de monitorar as emergências de saúde pública, de desencadear os planos de contenção, de manejar crises agudas e crônicas e de avaliar a situação de saúde, como atividades de contrainteligência epidemiológica por salvaguardas ativas ${ }^{9}$. Há também as medidas de defesa passiva, que promovem a segurança do alvo, atuando na antecipação dos eventos adversos, usando a metodologia de avaliação de risco ("risk assessment"), que considera a medida de probabilidade de um dado evento ocorrer e a gravidade dos danos, caso o evento ocorra. Nesta avaliação, os eventos raros ou que 
provoquem danos irrisórios não têm grande importância para as decisões estratégicas.

A avaliação de risco, ferramenta importante para a análise de inteligência, considera quatro etapas: (1) identificação do evento adverso ("hazard identification”), que se baseia na percepção do risco e no conhecimento dos potenciais eventos adversos, listando-os e classificando-os em ordem de importância ou de valor cultural; (2) a concepção do evento adverso ("risk assessment"), que pode utilizar métodos estocásticos de estimação, admitindo informações causais e casuais, ou utilizar protocolos de eventos sentinelas de perigo ambiental, químico, nuclear ou biológico, além da extensão de seus efeitos; (3) gerenciamento de evento adverso ("risk management"), que deve considerar, além dos referenciais científicos, as questões legais, econômicas, políticas e sociais, e (4) comunicação do evento adverso ("risk communication"), ao relatá-lo aos níveis decisórios superiores ${ }^{5}$.

A SVS é uma das cinco secretarias subordinadas ao Ministério da Saúde que tem por finalidades fomentar a captação de notificações, manejar e analisar dados, disseminar a informação, assessorar na tomada de decisões e desencadear ações direcionadas à proteção do estado de bem-estar, tornando-a, dessa forma, uma unidade de inteligência epidemiológica. Nesta Secretaria, está lotado o CIEVS, com as atribuições de monitorar a exatidão das fontes de dados em saúde e de promover a capacitação de recursos humanos ${ }^{10}$.

O conceito de emergência em saúde pública de interesse internacional surgiu com o Regulamento Sanitário Internacional, para identificar "um risco [ameaça] de saúde pública para outro Estado por meio da propagação internacional de doenças", que "requeiram uma resposta internacional coordenada". Este documento foi reconhecido por 194 países e pelos Estados-membros da Organização Mundial da Saúde e influenciou a atual concepção de inteligência epidemiológica. Tal Regulamento, após dez anos de discussão, foi implantado em junho de 2007 e enfocou a prevenção, a proteção, o controle das condições adversas à saúde pública (defesa passiva) e a coordenação de alertas e de respostas (defesa ativa). Passou-se, então, a considerar que as ameaças epidemiológicas não se limitam a uma lista de doenças ou de vias de transmissão, mas a um conjunto de condições que podem ameaçar o estado de bem-estar, incluindo a defesa globalizada da saúde, por alertas e respostas coordenadas contra epidemias e eventos acidentais ou mesmo deliberados, de origem biológica, quími- ca ou nuclear, destacando o respeito à dignidade humana, aos direitos humanos, às liberdades constitucionais e à universalidade das ações ${ }^{11,12}$.

O Center for Disease Control and Prevention (CDC) adota um critério bem definido para classificar as emergências em saúde pública: epidemias, emergências químicas, emergências radioativas, desastres naturais, fenômenos de massa e bioterrorismo ${ }^{13}$. Tal classificação é semelhante à adotada pela Secretaria Nacional de Defesa Civil (SEDEC), para identificar os desastres. O Centro Nacional de Gerenciamento de Riscos e Desastres (CENAD), sob a coordenação técnica do Departamento de Resposta aos Desastres e Reconstrução (DRD), diferencia os desastres de acordo com a evolução (agudo e crônico), a intensidade (acidentes, médio porte e muito grande porte) e a origem (natural, humana e misto). Resumidamente, os desastres de origem natural podem ser siderais (meteoros e corpos celestes), relacionados à geodinâmica terrestre (eólica, temperaturas extremas, precipitação hídricas, sismológica, vulcanológica, erosão do solo) e relacionados com desequilíbrios na biocenose (pragas animais e vegetais); os desastres humanos podem ser de natureza tecnológica (indústria, transportes, construção civil, incêndios, produtos perigosos, demográficos, energético e de outros recursos), de natureza social (ecossistema urbano e rural, convulsões sociais e conflitos bélicos) e de natureza biológica (doenças transmitidas por vetores biológicos, pela água e/ou alimentos, por inalação, pelo sangue ou secreções orgânicas e por outros mecanismos); os desastres mistos representam a combinação de mais de uma forma de desastre ${ }^{14}$. As definições de emergência em saúde pública e de desastres são muito semelhantes, podendo ser usadas sem distinção; note-se que são mais completas que uma lista de patologias.

As emergências em saúde pública ou os desastres, de qualquer origem, implicam prejuízos econômicos e sociais, principalmente, por afetar o estado de bem-estar, provocando o aumento da incidência de doenças e de mortes desnecessárias, a contaminação dos suprimentos de água e de alimentos, a destruição da infraestrutura de serviços, além dos efeitos negativos sobre o estado psicológico da comunidade, dentre outros efeitos ${ }^{15}$. Em todos os desastres naturais, ocorrem reações sociais de pânico e/ou de paralisação, advindas da movimentação de massas populacionais, organizadas e/ou não, das exposições às intempéries climáticas, da logística de distribuição de alimentos e de água potável, da diminuição de qualidade sanitária, do aumento de eventos em 
saúde mental, dos danos à infraestrutura dos serviços de saúde, além do aumento da incidência de doenças transmissíveis ${ }^{16}$. Uma catástrofe é um desastre de grandes proporções. Nestas situações, medidas de defesa ativa são desencadeadas, numa abordagem de atividade de contrainteligência restritiva, com a decretação de estado de defesa prevista na Constituição Federal.

\section{Geopolítica e salvaguardas internacionais}

A inteligência epidemiológica, tanto na abordagem estratégica quanto na restritiva, deve considerar as relações entre nações e suas influências nas políticas de saúde, usando uma lógica geopolítica, ou de geografia política, na medida em que existam pressões, ou demandas, do cenário internacional sobre o setor. A influência da Organização Mundial da Saúde (OMS), por exemplo, no cenário internacional é pautada pela manutenção de um estado de bem-estar global, focando o desenvolvimento humano, a segurança em saúde, os sistemas de saúde, a pesquisa científica e o ciclo de informação, além de compartilhar responsabilidades com os Estados Nacionais; por exemplo: a OMS coordena a Rede Global de Alerta e de Respostas (GOARN: Global Outbreak Alert \& Response Network), contando com a colaboração de instituições de pesquisa científica, governamentais ou não governamentais, oferecendo o suporte técnico e operacional aos países ao enfrentar emergências em saúde pública, desencadeando ações de respostas rápidas, investigando as ameaças de epidemias explosivas e criando um arranjo geopolítico focado nas emergências em saúde pública. A Organização Pan-Americana da Saúde é o braço da OMS nas Américas.

Por outro lado, a formação de blocos comerciais, como o Mercado Comum do Sul (Mercosul), com os objetivos de liberar o comércio de bens e de serviços, e de viabilizar a livre circulação de pessoas e de capitais, com a ampliação dos mercados nacionais e com o desenvolvimento econômico socialmente justo ${ }^{17}$, traz consequências. Assim, a intensificação da dinâmica humana nas fronteiras, nos pólos industriais e nos centros de distribuição de bens e serviços facilita a propagação de epidemias, sendo esta uma grande preocupação para a OMS. Há uma atenção especial sobre a porosidade da fronteira terrestre brasileira, por fazer divisa com todos os países da América do Sul, menos Equador e Chile, por onde ocorrem fluxos migratórios legítimos, ou não legítimos, com reflexos diretos e indiretos sobre o planejamento dos serviços de saúde, ge- rando condições de vulnerabilidade, de exclusão social e de agressões/violências. Para enfrentar estes quadros preocupantes, pactos são feitos, para organizar um sistema de informações e para desenvolver cooperações técnicas entre os países do Mercosul, contemplando a vigilância epidemiológica e o controle sanitário, em portos, aeroportos, terminais rodoviários e aduanas.

Tais preocupações ocorrem devido a uma possível, ou até iminente, pandemia de gripe, tão perigosa quanto a gripe espanhola de $1918^{18}$ possibilidade esta justificada pela degradação geográfica e pela manipulação genética. A degradação geográfica ${ }^{19}$ expropria recursos naturais e desaloja populações, expondo pessoas aos reservatórios naturais de doenças, que coexistiam em equilíbrio ecológico e que foram transformadas em ameaças ao estado de bem-estar, criando as condições para as pandemias de gripe aviária (H5N1), de doença respiratória severa aguda (SARS) e de gripe A (H1N1), além de expor as pessoas aos complexos patogênicos, pelos fluxos migratórios de indivíduos socialmente vulneráveis. A manipulação genética, que deriva dos desenvolvimentos científicos, da biologia moderna e da medicina, segundo Dando ${ }^{20}$, é importante para a pesquisa de vacinas e de antídotos, mas, também representa grande perigo, por ser capaz de produzir uma arma biológica ou provocar desastres biológicos, a partir dos mesmos recursos tecnológicos usados pela pesquisa científica: são as tecnologias de uso dual. As atividades de inteligência epidemiológica devem incluir a proteção do meio ambiente, o combate à degradação geográfica e a proteção do patrimônio genético e do conhecimento tradicional associado encontrado na Floresta Amazônica, no Cerrado, no Pantanal, na Mata Atlântica e nas reservas naturais distribuídas pelo território brasileiro, cuja legitimidade é reconhecida pela comunidade internacional. A Convenção de Diversidade Biológica (CDB) de 1992, que é reconhecida por 187 países signatários, chamou a atenção do mundo para o valor do patrimônio biológico e baseia-se no respeito à soberania de cada Estado-Nação sobre a biodiversidade de seus territórios ${ }^{21}$. Cumprindo a responsabilidade de proteger tal patrimônio, o Governo Brasileiro instituiu a Medida Provisória no 2.186-16 de 2001², para credenciar coleções de culturas microbiológicas junto ao Conselho de Gestão do Ministério do Meio Ambiente, como salvaguarda (defesa passiva) de tais recursos contra a biopirataria e contra a bioespionagem. As políticas de incentivo à segurança biológica de laboratórios, de biossegurança e de 
biosseguridade promovem a qualidade técnica dos Laboratórios Centrais dos Estados (LACEN), ampliando a capacidade de diagnóstico de doenças e aumentando a credibilidade técnica-científica em pesquisas envolvendo materiais de interesse estratégico ou de bens sensíveis ${ }^{12,23}$.

O Regulamento Sanitário Internacional enfoca as emergências em saúde pública causadas por material biológico, químico ou nuclear, ocorridas por acidente ou por ação deliberada. Tal documento representa um instrumento geopolítico de saúde, do qual o Brasil é signatário, que é incluído na pauta de negociações entre países; o País também é signatário dos principais tratados e convenções internacionais de não agressão e de não proliferação de material bélico, destacando a Convenção para Proibição de Armas Biológicas e Bacteriológicas (CPAB).

\section{Comunidade de inteligência epidemiológica}

No cenário da geopolítica de saúde, há uma variedade de situações que caracterizam ciclos de inteligência, em abordagens estratégicas e/ou restritivas, que podem identificar objetos, sujeitos, ameaças, fontes de informações (osint), atividades de inteligência e de contrainteligência etc. Tal variedade corrobora o entendimento de que a inteligência epidemiológica não se restringe, apenas, às ações e aos programas do SVS, mas extrapola competências e missões de órgãos governamentais ou não governamentais, em torno do estado de bem-estar de pessoas e de populações. A atividade de inteligência de epidemias é importante para a geopolítica de saúde, mas não reflete todas as especialidades da inteligência epidemiológica; se isto fizer sentido, pode ser considerado que a atividade de inteligência epidemiológica seja caracterizada pelo envolvimento das diferentes competências das secretarias do Ministério da Saúde, integradas a outros Ministérios, como sendo um caminho para a formação de comunidades de inteligência epidemiológica.

Para que se formem tais comunidades, é necessário organizar o ciclo de informação nas três esferas tripartite de governo, priorizando a universalidade da cobertura dos subsistemas de informações em saúde - do Datasus, por exemplo. Uma boa estratégia é investir na expansão da estrutura da Rede Interagencial de Informações para a Saúde (RIPSA), estimulando a criação de agências especializadas, em nível local, que seriam capazes de absorver os profissionais capacitados em programas de pós-graduação - acadê- mico ou profissional - nas áreas de saúde pública/saúde coletiva. A RIPSA, cumprindo um acordo de cooperação técnica firmado entre o Ministério da Saúde e a Organização Pan-Americana da Saúde, tem a finalidade de promover a integração entre agências especializadas em informação epidemiológica, contribuir para a viabilização do ciclo de informação, disponibilizar dados básicos e realizar análises em área relevantes para a saúde pública. Ao Datasus, compete organizar, validar e publicar os Indicadores e os Dados Básicos para a Saúde (IDB), além de outros produtos informacionais, seguindo as diretrizes das instâncias colegiadas e da RIPSA. Ambos têm papéis cruciais.

O aperfeiçoamento do ciclo de informação epidemiológica é necessário na esfera regional para subsidiar a gestão estratégica, os pactos de gestão do SUS, os planos de respostas rápidas ou emergenciais, a regulação médica, a integração horizontal e vertical de unidades de informação, as redes de atenção à saúde etc. As atividades de vigilância epidemiológica são bem estruturadas, mesmo que seja necessário aperfeiçoar processos do subsistema de informações; no entanto, a rede de atenção básica deve ser prioritária, pois representa uma rede de informações sentinela para os alertas e as respostas rápidas (humint), que podem alimentar subsistemas da Defesa Civil, por exemplo.

As salas de situação em saúde representam um instrumento de monitoramento de crises, ou de desastres, ou de epidemias, permitindo adotar "matrizes conceituais e marcos referenciais variados e até contraditórios" ${ }^{24}$; a implantação deste instrumento, em escala geográfica regional, representa um incremento estratégico no subsistema de inteligência, que poderia admitir contratos de gestão, incluindo agências privadas autenticáveis, certificáveis e auditáveis, formando uma rede descentralizada de informação em saúde (osint, masint, imint, sigint e humint). "A integração das ações promocionais, preventivas, curativas e reabilitadoras exige, inicialmente, o estabelecimento de uma população vivendo em um território. [...] A população de responsabilidade de um distrito sanitário deve ser esquadrinhada demográfica e epidemiologicamente e dividida em subgrupos [...] O processo de territorialização deve ser baseado em parâmetros espaciais e temporais" ${ }^{\prime 25}$.

Tais assertivas configuram uma inteligência epidemiológica, seguindo a abordagem estratégica, mas a abordagem restritiva também pode ser necessária, em situações de perigo iminente 
ou de catástrofes, cujas ações podem reduzir a autonomia de indivíduos, pela adoção de quarentenas, ou de barreiras sanitárias, ou de remoção de locais sobre risco, por exemplo. Entretanto, existem situações que merecem a atenção das autoridades, principalmente aquelas que envolvem tecnologias de uso dual. Tendo-se as emergências em saúde pública em perspectiva geopolítica e sem perder de vista as disputas e os conflitos internacionais, é necessário estabelecer códigos de conduta em biossegurança e biosseguridade para laboratórios que fazem pesquisa com bens sensíveis, sob o pretexto de proteger o estado de bem-estar e para assegurar que o desenvolvimento científico e tecnológico seja legítimo ${ }^{26}$.

Atlas e Repply ${ }^{27}$ sugerem que sistemas de biosseguridade efetivos devem controlar o acesso de pessoas aos ambientes onde ficam estocados ou onde são manipulados os agentes patogênicos. "O conhecimento, os materiais e a tecnologia necessários para construir e usar uma arma biológica são prontamente acessíveis pelo mundo. Os agentes patogênicos são encontrados na natureza por toda parte e podem ser acessíveis em hospitais, em laboratórios de pesquisa, em coleções de cultura, em pessoas e em animais doentes/portadores. As habilidades e equipamentos para produzir uma arma biológica são os mesmos que são necessários para desenvolver a medicina, a agricultura e outras áreas do conhecimento legítimo, de maneira que não deve impedir sua utilização. É possível, atualmente, sintetizar vírus a partir de componentes de organismos inativos/não-vivos, usando uma tecnologia que se torna cada vez mais barata e acessível" 28 .

Em um primeiro momento, pode parecer que tais medidas representam um obstáculo adicional às atividades de pesquisa ou um procedimento de intervenção na pesquisa científica, mas não se deve perder de vista que existem culturas beligerantes, de origem ideológica ou fundamentalista, que podem querer usar material biológico como instrumento de intimidação ou de destruição. O livro intitulado Contágio, do médico e novelista americano Robin Cook, publicado em 1995 - portanto anterior ao ataque ao World Trade Center -, relata uma história de óbitos em série, ocorridos em hospitais de New York, para atingir o AmeriCare, e mostra a vulnerabilidade da sociedade, como no seguinte trecho: "Sei o que está pensando, disse Laurie, balançando o dedo. A gripe era uma das doenças que você disse que usaria se fosse um terrorista tentando iniciar uma epidemia....'29. Assim, mesmo que a arte não imite a vida e vice-versa, o enredo fica impregna- do no ideário, no senso comum, com o agravante de que o conhecimento tecnológico disponível e a existência de coleções de agentes patogênicos multidroga-resistentes, de fácil acesso, possibilitam transformar a virulência de microorganismos e produzir organismos letais ${ }^{20,30,31}$. O País investe em pesquisa científica e tecnológica, e uma boa parte destas atividades é desenvolvida em universidades e em instituições de pesquisa ${ }^{32}$ que, também, promovem a capacitação profissional, em programas de pós-graduação lato sensu e stricto sensu, além de programas de iniciação científica em nível de graduação $0^{33}$. Em muitos destes centros, não existem controles efetivos sobre as dependências físicas e tampouco de proteção do conhecimento e dos serviços terceirizados (limpeza e segurança patrimonial), viabilizando as ameaças externas, as atividades de inteligência competitiva (mercado) ou o bioterrorismo.

Furtado $^{34}$ relacionou vários agentes patogênicos de uso dual, que podem ser encontrados, com certa facilidade, no País e que integram a lista de bens sensíveis do Ministério de Ciência e Tecnologia (Resolução CIBES no 8/2007), como bactérias, ricketsias, fungos, viruses/príons, protozoários e nematoides. Dentre estes, destacamse o Mycobacterium tuberculosis, o Mycobacterium bovis, a Yersinia pestis, o Vibrio cholera, as Salmonellas, a Francisella tularensis, a Histoplasma capsulatum, a Herpesvirus, Retroviruses, o Vírus da febre amarela, o Vírus da febre hemorrágica do dengue e o Vírus da varíola humana. Para Lima ${ }^{35}$, com o final da guerra fria e a globalização, vários profissionais de inteligência foram absorvidos pela inteligência competitiva; o mesmo ocorreu no Brasil, com a criação de empresas que oferecem cursos e serviços de inteligência corporativa e empresarial ${ }^{36}$. Portanto, este é um assunto que merece ser tratado com atenção.

O presente trabalho adaptou o conceito de inteligência epidemiológica ao referencial teórico do modelo epidemiológico de campo de saúde, que tem a organização dos serviços de saúde como um importante fator determinante/condicionante das emergências em saúde pública. A existência de geopolíticas de saúde torna a inteligência epidemiológica uma atividade de Estado, considerando o estado de bem-estar de pessoas, ou de populações, um direito constitucional a ser defendido/protegido. Tal atividade de inteligência deve ser construída coletivamente, tendo a Universidade o papel de ativador, contribuindo com a formação de recursos humanos, a análise de risco, a elaboração de planos estratégicos, a pesquisa operacional, o suporte técnico à toma- 
da de decisões etc. Contribuições estas que visem à integração da vigilância em saúde, da atenção básica, da pesquisa científica, da capacitação de pessoal, além de interagir com os vários setores da sociedade, para construir um modelo de atuação em emergências/crises compatível com os valores democráticos.

\section{Referências}

1. Waldman EA. A vigilância como instrumento de saúde pública. In: Waldman EA, organizador. Vigilância em saúde pública. São Paulo: Faculdade de Saúde Pública da Universidade de São Paulo; Série Saúde \& Cidadania; 1998. p.91-131.

2. Santos JLF, Westphal MF. Práticas emergentes de um novo paradigma de saúde: o papel da universidade. Estudos Avançados 1999; 13(35):71-88.

3. Centro Franco-Brasileiro de Documentação Técnica. Inteligência estratégica. In: Seminário internacional Inteligência Estatégica; 2007; São Paulo, Rio de Janeiro, Curitiba.

4. Menezes JG, Marcial EC. A inteligência competitiva e o quarto canal. In: Anais do II Workshop Brasileiro de Inteligência Competitiva e Gestão do Conhecimento; 2001; Florianópolis. p.1-14.

5. North DW. Limitations, definitions, principles and methods of risk analysis. Rev Sci Tech Off Int Epiz 1995; 14(4):913-923.

6. Tarapanoff K, Araújo-Jr RH, Cornier PMJ. Sociedade da informação e inteligência em unidades de informação. Ci Inf 2000; 29(3):91-1000.

7. Cepik M. Inteligência e políticas pública: dinâmicas operacionais e condições de legitimação. Security and Defense Studies Review 2002; 2(2):246-267.

8. Brasil. Decreto no 4.376 de 13 de setembro de 2002 . Dispõe sobre a organização e o funcionamento do Sistema Brasileiro de Inteligência, instituído pela Lei no 9.883, de 7 de dezembro de 1999, e dá outras providências. Diário Oficial da União 2002; 16 set.

9. Brasil. Ministério da Saúde. Secretaria de Vigilância em Saúde. Vigilância em saúde no SUS: fortalecendo a capacidade de resposta aos velhos e novos desafios. Brasília: Ministério da Saúde (MS); 2006.

10. Brasil. Portaria no 30 de $7 / j u l / 2005$. Institui o Centro de Informações Estratégicas em Vigilância em Saúde, define suas atribuições, composição e coordenação. Diário Oficial da União 2005; 8 jul.
11. World Health Organization (WHO). International health regulations. $2^{\text {nd }}$ ed. Geneve: World Health Organization (WHO); 2005.

12. Carmo EH, Penna G, Oliveira WK. Emergências de saúde pública: conceito, caracterização, preparação e resposta. Estudos Avançados 2008; 22(64):19-32.

13. Allen H, Katz R. Demography and public health emergency preparedness: making the connection. Popul Res Policy Rev 2010; 29(4):527-539.

14. Brasil. Ministério da Integração Nacional (MIN). Secretaria Nacional de Defesa Civil. Política nacional de defesa civil. Brasília: Ministério da Integração $\mathrm{Na}-$ cional (MIN); 2007. [acessado 2012 fev 8]. Disponível em: http://www.defesacivil.gov.br/download/ download.asp?endereco=/publicacoes/publicacoes/ pndc.pdf\&nome_arquivo=pndc.pdf.

15. Tekeli-Yesil S. Public health and natural disasters: disaster preparedness and response in health systems. J Public Health 2006; 14(5):317-324.

16. Pan American Health Organization (PAHO). Natural disasters: protecting the public's health. Washington: Pan American Health Organization (PAHO); 2006.

17. Brasil. Ministério da Saúde. A saúde no Mercosul. $3^{a}$ ed. Brasília: Ministério da Saúde (MS). 2004.

18. Ujvari SC. A história da disseminação dos microrganismos. Estudos Avançados 2008; 22(64):171-182.

19. Peiter PC. A geografia da saúde na faixa de fronteira continental do Brasil na passagem do milênio [tese]. Rio de Janeiro: Instituto de Geociências da Universidade Federal do Rio de Janeiro; 2005.

20. Dando M. The impact of the development of modern biology and medicine on the evolution of offensive biological warfare programs in the Twentieth Century. Defense Analysis 1999; 15(1):43-62. 
21. Araújo NL, Leal AF, Oliveira DA. Conhecimentos tradicionais e patrimônio cultural imaterial: formas de proteção. Montes Claros: Universidade Estadual de Montes Claros; 2006.

22. Brasil. Medida Provisória no 2.186-16 de 2001. Regulamenta o inciso II do $\$ 1$ o e o $\$ 4$ o do art. 225 da Constituição, os arts. 1o, 8o, alínea "j", 10, alínea "c", 15 e 16, alíneas 3 e 4 da Convenção sobre Diversidade Biológica, dispõe sobre o acesso ao patrimônio genético, a proteção e o acesso ao conhecimento tradicional associado, a repartição de benefícios e o acesso à tecnologia e transferência de tecnologia para sua conservação e utilização, e dá outras providências. Diário Oficial da União 2001. 24 ago.

23. Brasil. Lei no 9.112 de 10 de outubro de 1995. Dispõe sobre a exportação de bens sensíveis e serviços diretamente vinculados. e serviços diretamente vinculados. Diário Oficial da União 1995; 11 de out. [acessado 2012 fev 8]. Disponível em: http://www.mct.gov.br/ index.php/content/view/5291.html.

24. Vasconcellos MM. Sala de situação em saúde: contribuição à ampliação da capacidade gestora do Estado? In: Organização Pan-Americana da Saúde (Opas). Sala de situação em saúde: compartilhando experiências do Brasil. Brasília: Organização PanAmericana da Saúde (Opas), Ministério da Saúde (MS); 2010.

25. Mendes EV. Revisão bibliográfica sobre redes de atenção à saúde. Belo Horizonte: Secretaria de Estado de Saúde de Minas Gerais; 2007. [site na Internet]. Disponível em http://portal.saude.gov.br/portal/arquivos/pdf/ revisao_bibliografica_redes.pdf.

26. Cardoso TAO, Navarro MBMA, Soares BEC, Tapajós AM. Biosseguridade e biossegurança: aplicabilidades da segurança biológica. Interciência 2008 33(8):561-568.
27. Atlas RM, Reppy J. Globalizing biosecurity. Biosecurity and Bioterrorism: Biodefense Strategy, Practice, and Science 2005; 3(1):51-60.

28. Gronvall GK. Preventing the development and use of biological weapons. Biosecurity and Bioterrorism: Biodefense Strategy, Practice, and Science 2009; 7(1):3132.

29. Cook R. Contágio. Rio de Janeiro: Bestbolso; 2008.

30. Parnell GS, Borio LL, Brown GG, Banks D, Wilson AG. Scientists urge DHS to improve bioterrorism risk assessment. Biosecurity and Bioterrorism: Biodefense Strategy, Practice, and Science 2008; 6(4):353-357.

31. Tutunji V. Guerra biológica: uma revisão. Universitas Ciências da Saúde 2002; 1(1):105-139.

32. Chaimovich H. Biosseguridade. Estudos Avançados 2005; 19(55):261-269.

33. Trindade JCS, Pringezi LS. Instituições universitárias e produção do conhecimento. São Paulo em Perspectiva 2002; 16(4):9-14.

34. Furtado STF. Bioterrorismo. Uberlândia: EDUFU; 2008.

35. Lima AVF. O papel da inteligência na atualidade [monografia] Brasília: Faculdade Albert Einstein; 2004.

36. Freud, o agente 0013. Revista Veja 2010; 5 maio: 82.

Apresentado em 22/10/2010

Aprovado em 06/01/2011

Versão final apresentada em 13/01/2011 